\title{
Navigation in der Neurochirurgie
}

\author{
Günther C. Feigl, Marcos Tatagiba
}

\section{Zusammenfassung}

Die Hauptaufgabe der Neuronavigation in der Neurochirurgie ist es, den Neurochirurgen dabei $\mathrm{zu}$ unterstützen, eloquente Strukturen in einer durch pathologische Läsionen veränderten Anatomie leichter und schneller zu lokalisieren. Die Neuronavigation basiert auf MRT-, CT- oder Ultraschallbildgebung und ist vergleichbar mit einem GPS-System für das zentrale Nervensystem. Das Haupteinsatzgebiet ist die Hirntumorchirurgie und die spinale Neurochirurgie. Die Verfügbarkeit von Neuronavigationssystemen hat es ermöglicht, dass Neurochirurgen Hirntumoren über minimalinvasive Zugänge entfernen und durch die erhöhte Sicherheit bessere Operationsergebnisse erzielen können. Auch die Genauigkeit der Platzierung von Pedikelschrauben bei Wirbelsäulenstabilisierungen ist durch den Einsatz dieser Systeme signifikant erhöht und führt zu besseren operativen Ergebnissen. Die Neuronavigation kann aber fehlendes anatomisches Wissen bzw. fehlende operative Erfahrung nicht ausgleichen.

\section{Computer-Assisted Navigation in Neurosurgery}

The main purpose of the available navigation systems is to support the neurosurgeon in localising delicate anatomic structures in regions of the central nervous systems which are altered due to lesions such as brain tumours. The neuronavigation system also enables the neurosurgeon to remove tumours through minimally invasive craniotomies. The navigation systems use MRI and CT as well as ultrasound images and are comparable to a GPS system for the central nervous system. The neuronavigation system is mainly used for brain tumour surgery and spine surgery. Using these systems, surgical results can be improved and the placement accuracy of pedicular screws for stabilisation of the vertebral column is significantly better, leading to better operative outcome. However, a lack of anatomic knowledge and surgical experience cannot be compensated for by using a neuronavigation system.

\section{Einleitung}

Bereits im Jahr 1908 wurden von Sir Victor Horsley und Robert H. Clarke mit der Entwicklung des Horsley-Clarke-Apparatus die ersten Versuche unternommen, ein Navigationssystem für neurochirurgische Eingriffe zu entwickeln. Trotz der eingeschränkten Möglichkeiten in der Bildgebung konnten so die ersten stereotaktischen Systeme entwickelt werden und wurden dann in den 40er-

OP-JOURNAL 2011; 27: 118-122

(c) Georg Thieme Verlag KG Stuttgart · New York DOI http://dx.doi.org/10.1055/s-0031-1280184

Jahren des 20. Jahrhunderts von Ernest A. Spiegel und Henry T. Wycis in den USA wie auch von Lars Leksell in Schweden für Operationen am Gehirn eingesetzt. Mit der technologischen Weiterentwicklung der Bildgebung mit Computertomografie und Magnetresonanz als auch durch die Weiterentwicklung der Computertechnologie wurden dann in den 90er-Jahren des 20. Jahrhunderts die ersten Neuronavigationssysteme entwickelt.

Prinzipiell muss man eine rahmenbasierte und eine rahmenlose Navigation unterscheiden. Während die rahmenbasierte Navigation mit stereotaktischen Systemen durchgeführt wird, wo anhand vorhandener Bilder und eines Referenzrahmens die genauen Koordinaten eines Zielpunkts berechnet werden, werden bei der rahmenlosen Navigation ein Pointer (Zeiger) und im Navigationssystem registrierte Instrumente frei bewegt. Beide Arten der Navigation dienen demselben Zweck und zwar der präzisen Lokalisation von anatomischen Strukturen, v.a. in einer Umgebung, die eine durch pathologische Läsionen oder Traumen veränderte Anatomie aufweist. Die Navigation stellt so ein Werkzeug für den Neurochirurgen dar, welches die Orientierung auf kleinstem Raum millimetergenau ermöglicht. Des Weiteren ist die Navigation ein hilfreiches Instrument, um neurochirurgische Behandlungen über minimalinvasive Zugänge durchzuführen und so das operative Trauma und die Behandlungsergebnisse zu verbessern. Das Grundprinzip der Neuronavigation für den Neurochirurgen ist mit dem GPS-System eines Autos vergleichbar, bei dem der Patient das Gelände ist, auf dem sich der Neurochirurg mit dem Pointer bewegt und die Position auf der „Landkarte des Patienten“, dem CT- oder MRT-Bild, dargestellt wird.

Die Hauptaufgabe der Neuronavigation in der Neurochirurgie ist es, den Neurochirurgen dabei zu unterstützen, eloquente Strukturen in einer durch pathologische Läsionen veränderten Anatomie leichter und schneller zu lokalisieren.

\section{Arten und Funktionsweisen der Navigationssysteme}

Die Neuronavigation basiert auf MRT-, CT- oder Ultraschallbildgebung und ist vergleichbar mit einem GPS-System für das zentrale Nervensystem.

\section{Rahmenbasierte Navigation}

Die rahmenbasierte Navigation wird in der Neurochirurgie für stereotaktische bzw. für funktionelle Eingriffe einge- 


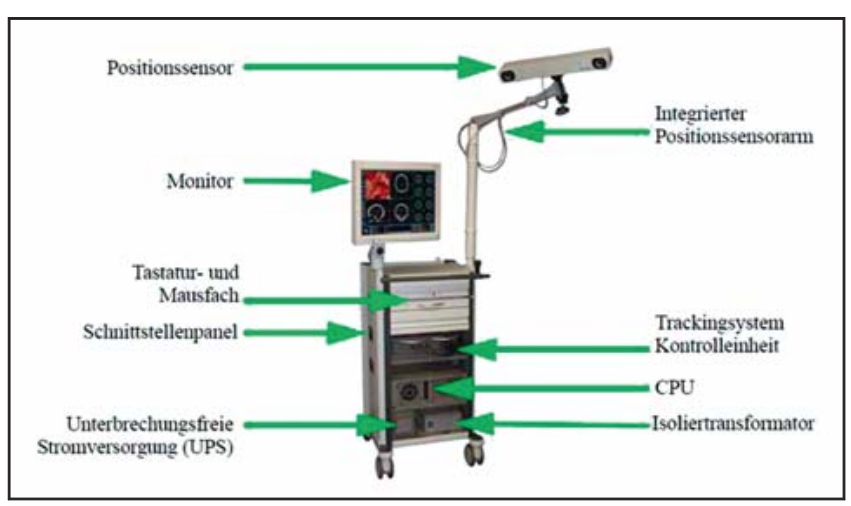

Abb. 1 Cbyon-Neuronavigationssystem.

setzt. Bei der Anwendung von stereotaktischen Navigationssystemen wird dem Patienten präoperativ ein Stereotaxiering an den Schädel geschraubt, mit dem dann unter Verwendung eines Referenzrahmens die präoperative Bildgebung durchgeführt wird. Mit den gewonnenen Bilddaten können so die Koordinaten des Zielpunkts millimetergenau berechnet werden. Der große Vorteil der stereotaktischen Eingriffe ist, dass diese Operationen in der Regel unter Lokalanästhesie durchgeführt werden können. Eine stereotaktische Biopsie (Nadelbiopsie) wird über ein kleines Bohrloch im Schädel durchgeführt. Das Hauptanwendungsgebiet hier ist die Gewinnung von Gewebsmaterial zur Bestimmung der Pathohistologie, um die weitere Therapie planen zu können. Dies wird hauptsächlich bei Patienten durchgeführt, bei denen eine Tumorentfernung nicht infrage kommt. Um die ideale Stelle für eine solche Tumorentnahme $\mathrm{zu}$ bestimmen, stehen verschiedene Bildgebungsmodalitäten zur Verfügung, wie z. B. die Spektroskopie oder das „Chemical Shift Imaging“ (CSI), anhand derer der optimale Zielpunkt mit der höchsten Gewebsaktivität des Tumors dargestellt werden kann. Eine weitere Einsatzmöglichkeit für die stereotaktische Navigation ist z.B. die Entleerung von Hirnabszessen oder Zysten.

Prinzipiell muss man eine rahmenbasierte und eine rahmenlose Navigation unterscheiden.

Ein weiterer, sehr großer Einsatzbereich der stereotaktischen Navigation ist die funktionelle Neurochirurgie, wie z.B. die Behandlung von Parkinson [3,7], wo wiederum in Lokalanästhesie Elektroden in den subthalamischen Nukleus eingeführt werden, um so die Krankheit durch elektrische Pulse, die über einen Hirnschrittmacher generiert werden, zu behandeln.
Für die o.g. Einsatzbereiche ist die stereotaktische Navigation sehr wertvoll, jedoch besteht der Nachteil dieser rahmenbasierten Navigation darin, dass die Instrumente nur über ein kleines Bohrloch und am Rahmen fixiert in das Gehirn eingeführt werden können und so eine freie Bewegung der Instrumente z.B. zur Entfernung von Tumoren nicht möglich ist.

Die rahmenbasierte Navigation wird in der Neurochirurgie für stereotaktische bzw. für funktionelle Eingriffe eingesetzt.

\section{Rahmenlose Navigation}

In der rahmenlosen Navigation, die in der Neurochirurgie eingesetzt wird, unterscheidet man zwischen den optischen und den elektromagnetischen bzw. Hybridsystemen. Bei der rahmenlosen Navigation können die benötigten Instrumente frei bewegt werden und so nicht nur präoperativ bestimmte Koordinaten lokalisiert, sondern jede beliebige Struktur intraoperativ lokalisiert werden. Ein optisches Neuronavigationssystem besteht aus einer Infrarotkamera, einem Referenzstern, einem Pointer und einem PC zur Verarbeitung der Information (Abb. 1). Wie auch bei der rahmenbasierten Navigation benötigt die rahmenlose Navigation eine Bildgebung, die in der Regel präoperativ in einem CT oder MRT durchgeführt wird. Je nach System können die Bilder für den intraoperativen Einsatz auch fusioniert werden, sodass bei Bedarf entweder das CT- oder MRTBild für die Navigation zur Verfügung steht. Bei den rahmenlosen Systemen muss der Patient im Navigationssystem registriert werden, was in den meisten Fällen mit einer punktbasierten Registrierung passiert. Hierfür werden präoperativ sog. „Fiducials“ (kleine röntgendichte Aufkleber) auf den Kopf des Patienten geklebt und dienen hier als definierte Punkte, anhand welcher der
Patient dann im Neuronavigationssystem registriert wird. Nach Registrierung des Patienten kann dann mithilfe des Pointers jede Struktur am Schädel, im Gehirn oder an der Wirbelsäule millimetergenau lokalisiert werden. Bei Bedarf können auch andere chirurgische Instrumente im Neuronavigationssystem registriert und so navigiert im OPSitus eingesetzt werden. Dies ist v.a. in der spinalen Neurochirurgie der Fall, wo Neuronavigationssysteme häufig für die Platzierung von Pedikelschrauben eingesetzt werden. Der große Vorteil der optischen rahmenlosen Navigationssysteme ist das große Ausleuchtungsfeld der Infrarotkamera und somit die fast uneingeschränkte Bewegung des Pointers im OP-Situs. Ein Nachteil der optischen Systeme ist die Tatsache, dass nur rigide Instrumente mit einer definierten Spitze und einer definierten Länge eingesetzt werden können.

In der rahmenlosen Navigation, die in der Neurochirurgie eingesetzt wird, unterscheidet man zwischen den optischen und den elektromagnetischen bzw. Hybridsystemen.

Bei den elektromagnetischen Systemen unterscheidet sich das Funktionsprinzip von den optischen Systemen nur dadurch, dass hier anhand einer elektromagnetischen Spule ein Magnetfeld im Bereich des OP-Situs aufgebaut wird und dieses Magnetfeld durch kleine Spulen in den Instrumentenspitzen gestört wird. Diese Störung wird im Computer millimetergenau berechnet und stellt so die Lokalisation des Pointers bzw. des Instruments im OP-Situs am Navigationssystem dar. Der Einsatzbereich der elektromagnetischen Systeme unterscheidet sich nicht wesentlich von dem der optischen Navigationssysteme. Ein großer Vorteil der elektromagnetischen Systeme ist die Tatsache, dass Instrumente bei Bedarf gebogen werden können und die Spitze trotzdem immer genau dargestellt werden kann. Nachteile der elektromagnetischen Systeme sind die Störanfälligkeit durch ferromagnetische Halterungen und Instrumente im OP-Situs und das kleine Magnetfeld, das durch diese Systeme generiert wird. Wenige Systeme sind als Hybridsystem verfügbar, wo zwischen dem optischen und dem elektromagnetischen System hinund hergeschaltet werden kann. 


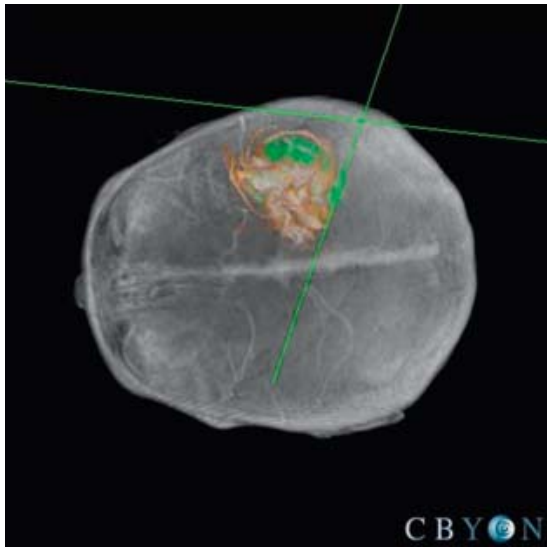

Abb. 2 Voxelbasierte 3-D-Darstellung aus dem Cbyon-Neuronavigationssystem. Die Transparenzänderung der Gewebeschichten ermöglicht es, in der 3-D-Rekonstruktion in den Schädel des Patienten „hineinzuschauen“.

\section{3-D-voxelbasierte Systeme}

Die meisten Neuronavigationssysteme am Markt, welche heutzutage in der Neurochirurgie eingesetzt werden, bieten 3-D-Rekonstruktionen der Bilddaten an, jedoch handelt es sich hier um ein sog. „Surface Rendering“, bei dem nur die Oberfläche als 3-D-Rekonstruktion dargestellt wird. Die modernsten Systeme verfügen jedoch über ein sog. 3-Dvoxelbasiertes Volume Rendering, bei dem nicht nur anhand der triaxialen Bilddaten (axial, koronar und sagittal) navigiert werden kann, sondern auch anhand der Voxel (3-dimensionales Bildpixel). Ein solches System bietet die Firma MedSurgical (Sunnyvale, CA, USA) mit dem Cbyon-System an. Mithilfe des Cbyon-Systems können Gewebeschichten im Navigationssystem transparent gemacht werden und dem Neurochirurgen wird es ermöglicht, anhand einer virtuellen endoskopischen Darstellung mithilfe des Pointers in den Schädel des Patienten zu schauen (Abb. 2). Mit dieser Funktion können präoperativ segmentierte wichtige anatomische Strukturen und pathologische Läsionen in der 3-DAnsicht (Abb. 3) leicht lokalisiert werden. Die voxelbasierte 3-D-Neuronavigation ermöglicht es auch, navigierte endoskopische Eingriffe hier v.a. an der Schädelbasis durchzuführen, da es möglich ist, parallel auf einem Bildschirm sowohl das „Live“-Endoskopievideobild als auch die virtuelle 3-D-Rekonstruktion parallel darzustellen.

\section{Einsatzbereiche der Neuronagivation}

Die Hauptaufgabe der Neuronavigation in der Neurochirurgie ist es, den Neurochirurgen dabei $\mathrm{zu}$ unterstützen, eloquente Strukturen in einer durch pathologische Läsionen veränderten Anatomie leichter und schneller zu lokalisieren. Gleichzeitig ermöglicht der Einsatz der Navigation auch die Durchführung von neurochirurgischen Eingriffen über minimalinvasive Zugänge. Die Neuronavigation kann aber fehlendes anatomisches Wissen bzw. fehlende operative Erfahrung nicht ausgleichen.

\section{Hirntumorchirurgie}

Bei der Resektion von Hirntumoren wird die Neuronavigation v.a. bei der Resektion von Tumoren in eloquenten Arealen eingesetzt, wo anhand der entsprechenden MR-Bildgebung (fMRI = funktionelle Magnetresonanztomografie und DTI = Hirnbahndarstellung) die operativen $\mathrm{Zu}$ gänge so geplant werden können, dass Funktionsareale nicht verletzt werden $[1,2]$. Ein limitierender Faktor der Neuronavigation ist die Tatsache, dass die Navigation in der Regel auf präoperativ

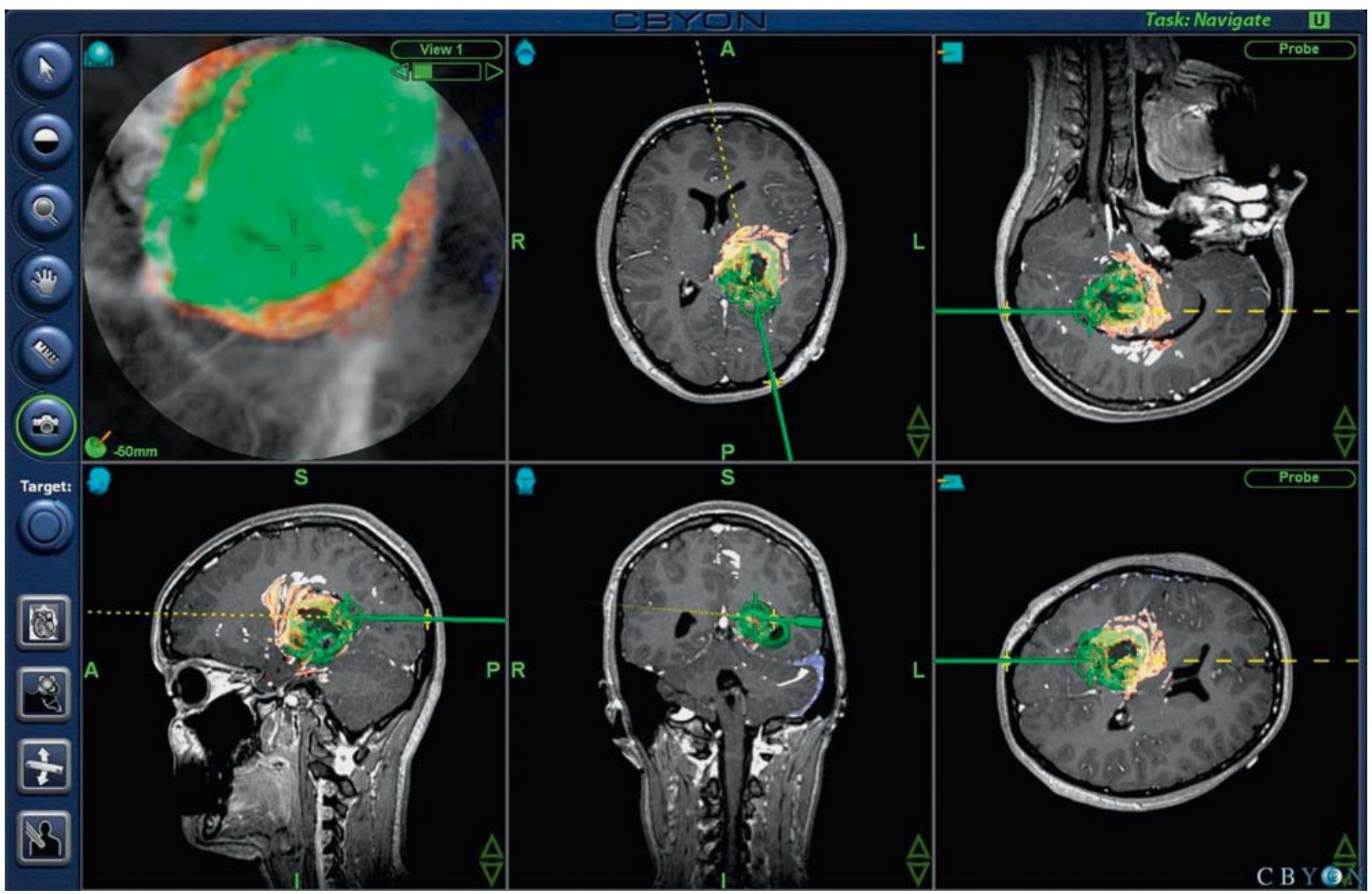

Abb. 3 Voxelbasierte 3-D-Darstellung aus dem Cbyon-Neuronavigationssystem. Im linken oberen Bild sieht man die virtuelle Endoskopieansicht (Tumor grün, Hirnbahnen orange). Man erkennt, wie der Tumor die Hirnbahnen auseinanderdrängt. 


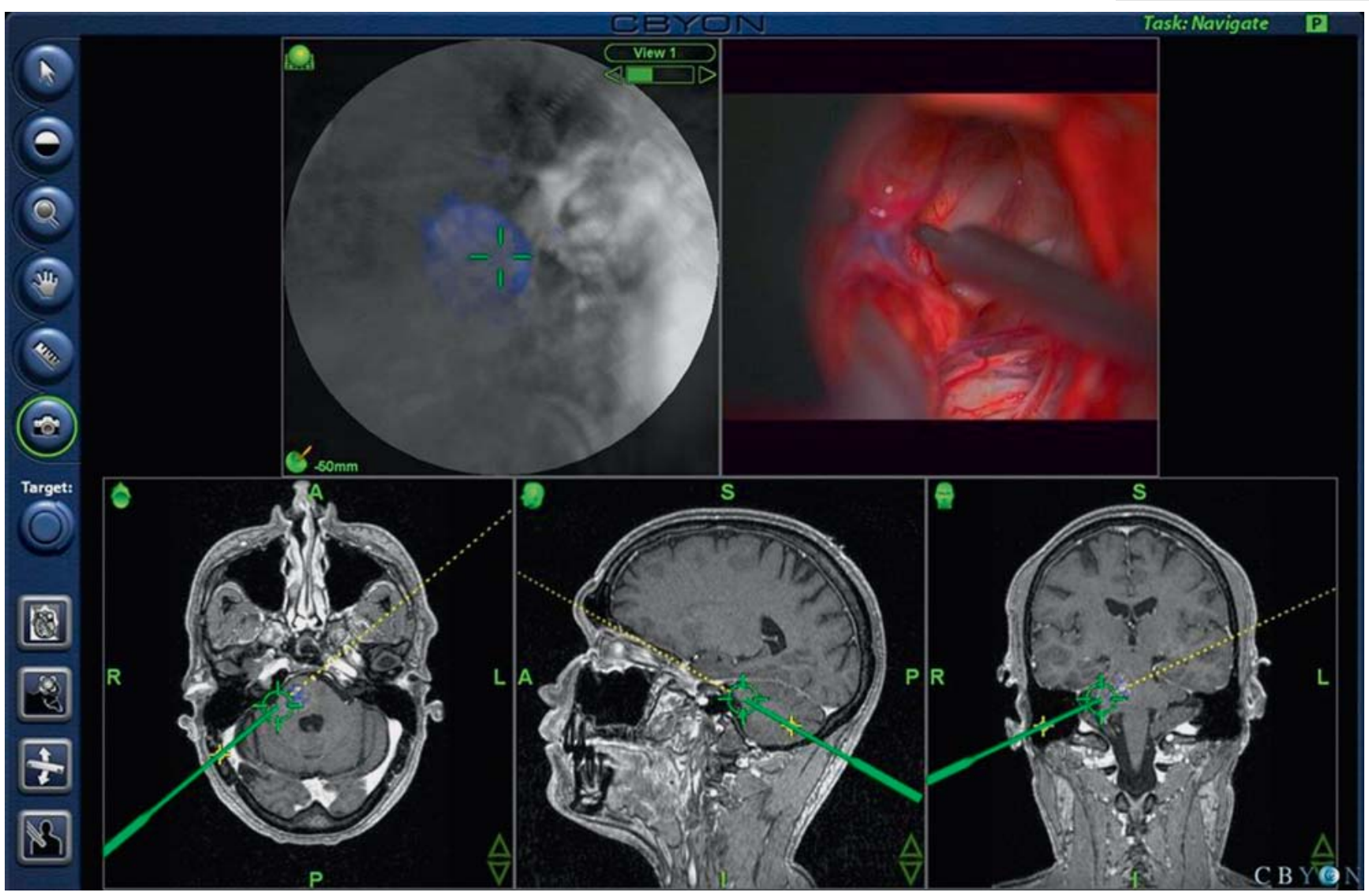

Abb.4 Intraoperative Navigationsbilder aus dem Cbyon-Neuronavigationssystem. Im linken oberen Bild ist das Kavernom in der virtuellen 3-DDarstellung durch die Transparenzänderung der Gewebeschichten sichtbar (blau). Rechts daneben sieht man das Livebild aus dem Operationsmikroskop, bei dem die Pointerspitze die Lokalisation des Kavernoms am Hirnstamm zeigt.

aufgenommenen CT- oder MR-Bildern [4] basiert und somit eine Veränderung des OP-Situs nicht dargestellt wird. So tritt z. B. ein sog. „Brain Shift“ (Verlagerung des Gehirns) nach Eröffnung der Dura auf, da Liquor abläuft und sich die Position des Hirngewebes ändert. Es ergibt sich so eine Ungenauigkeit, die der Neurochirurg beim Einsatz der Neuronavigation immer in Betracht ziehen muss. Die Möglichkeit, dies zu umgehen, ist einerseits der Einsatz eines navigierten Ultraschalls [5,6], wie diese von der Fa. Sonowand (Trondheim, Norwegen) und BrainLab (Feldkirchen) angeboten werden, oder durch den Einsatz eines intraoperativen MRTs oder CTs, wo in kritischen Phasen der Operation die Bildgebung aktualisiert werden kann und somit auch die Genauigkeit der Neuronavigation gewährleistet wird. Hier besteht aber v.a. in Bezug auf die Anschaffungskosten und die Erhaltungskosten ein sehr großer Unterschied, wobei intraoperative MRT-Systeme zwar die beste Bildqualität für die Navigation liefern, jedoch auch das System mit den höchsten Anschaffungs- und Erhaltungskosten darstellen.

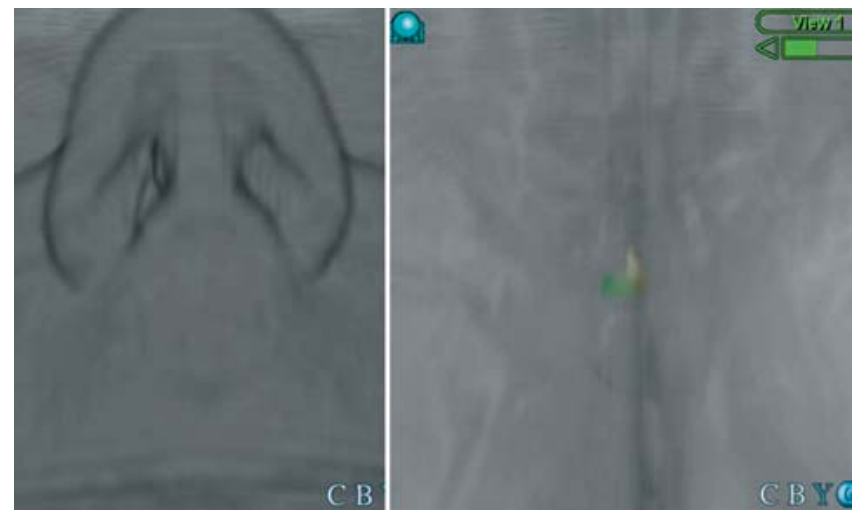

Abb. 5 Im linken Bild sieht man die virtuelle endoskopische Ansicht ohne Änderung der Transparenz der Gewebeschichten. Im rechten Bild werden durch die geänderte Transparenz der präoperativ segmentierte Hypophysenstiel (gelb) und der Hypophysentumor (grün) deutlich sichtbar.

Bei der Resektion von Hirntumoren wird die Neuronavigation v. a. bei der Resektion von Tumoren in eloquenten Arealen eingesetzt, wo anhand der entsprechenden MR-Bildgebung ( $\mathrm{fMRI}$ = funktionelle Magnetresonanztomografie und DTI = Hirnbahndarstellung) die operativen Zugänge so geplant werden können, dass Funktionsareale nicht verletzt werden.

Durch das Importieren der Bildgebung mit Darstellung der Funktionsareale und Hirnbahnen (Abb. 3) wird die intraoperative Lokalisation dieser Strukturen sehr erleichtert und somit die Sicherheit der Tumorresektion erheblich erhöht, was wiederum das operative Ergebnis verbessert. Auch für Operationen am Hirnstamm hat die Neuronavigation eine große Bedeutung, hier z.B. für die Resektion von Kavernomen, wo oft der Eintrittspunkt zur Läsion an der Oberfläche des Hirnstamms nicht immer eindeutig sichtbar ist (Abb.4). Da Nervenstimulatoren für das Brainmapping bzw. Mapping des Hirnstamms im Neuronavigationssystem registriert werden können, kann so gezielt der beste Eintrittspunkt 
in den Hirnstamm gewählt werden, um das Kavernom ohne Schäden am Hirnstamm entfernen zu können. Auch in der Schädelbasischirurgie wird die Neuronavigation häufig eingesetzt, wo z.B. im Bereich der vorderen Schädelbasis die Lokalisation des N. opticus, in der mittleren Schädelgrube die Lokalisation von Strukturen wie der A. carotis interna, dem Hypophysenstiel (Abb.5) oder des Foramen ovale für Rhizotomien bei Trigeminusneuralgien erheblich erleichtert wird.

Ein limitierender Faktor der Neuronavigation am Gehirn ist die Tatsache, dass die Navigation in der Regel auf präoperativ aufgenommenen CT- oder MR-Bildern basiert und somit eine Veränderung des OP-Situs (z. B. ein sog. „Brain Shift" nicht dargestellt wird.

Durch den Einsatz der Neuronavigation können Pedikelschrauben bei Wirbelsäulenstabilisierungen in allen Segmenten der Wirbelsäule (HWS, BWS und LWS) mit einer signifikant höheren Genauigkeit platziert werden und die Strahlenbelastung für Patienten und für das OPPersonal können erheblich verringert werden.

\section{Spinale Chirurgie}

Auch für die spinale Neurochirurgie hat die Neuronavigation einen hohen Stellenwert. Durch den Einsatz dieser Systeme können Pedikelschrauben bei Wirbelsäulenstabilisierungen in allen Segmenten der Wirbelsäule (HWS, BWS und LWS) mit einer signifikant höheren Genauigkeit, als dies frei Hand möglich ist, platziert werden. Mit sog. „2-D- bzw. 3-D-Fluorosystemen" kann auch die Strahlenbelastung für den Patienten und für das OP-Personal durch den Einsatz der Neuronavigation erheblich verringert werden, da nur eine Aufnahme vor Beginn der Operation nötig ist, um die Wirbelsäule des Patienten im Navigationssystem zu registrieren. In diesen
Fällen ist das sonst übliche Röntgen zur Kontrolle der Eintrittspunkte und der Lage der einzelnen Pedikelschrauben nicht mehr notwendig. Es sind mittlerweile auch schon navigationsgesteuerte Robotersysteme wie z.B. das „Spine Assist-System“ (Mazor Robotics, Israel) verfügbar, mithilfe dessen ein Roboter anhand von präoperativ geplanten Trajektorien die Platzierung der Pedikelschrauben unterstützt und eine Genauigkeit der gesetzten Pedikelschrauben von $98 \%$ ermöglicht.

Die Neuronavigation kann fehlendes anatomisches Wissen bzw. fehlende operative Erfahrung nicht ausgleichen.

\section{Schlussfolgerung}

Die Verfügbarkeit von Neuronavigationssystemen hat es ermöglicht, dass Neurochirurgen Hirntumoren über minimalinvasive Zugänge entfernen und durch die erhöhte Sicherheit bessere Operationsergebnisse erzielen können. Auch die Genauigkeit der Platzierung von Pedikelschrauben bei Wirbelsäulenstabilisierungen ist durch den Einsatz dieser Systeme signifikant erhöht und führt zu besseren operativen Ergebnissen. Neuronavigationssysteme sind jedoch nicht in der Lage, fehlendes anatomisches Wissen auszugleichen und so aus einem schlechten Neurochirurgen einen guten zu machen. Die Grenzen der Navigation stellen einerseits die Auflösung der verfügbaren Bildgebung und die Tatsache dar, dass die Navigation auf „Offline-Daten" basiert, welche sich nicht laufend an den OP-Situs anpassen und so im Laufe einer OP ihre Genauigkeit verlieren. Ein Lösungsansatz für diese Probleme sind einerseits die navigierten Ultraschallgeräte, die ein häufiges Aktualisieren der Navigation intraoperativ ermöglichen, oder intraoperative MRT- bzw. CTSysteme. Hier besteht v.a. in Bezug auf die Anschaffungskosten und die Erhaltungskosten ein sehr großer Unter- schied, wobei intraoperative MRT-Systeme zwar die beste Bildqualität für die Navigation liefern, jedoch auch das System mit den höchsten Anschaffungsund Erhaltungskosten darstellen.

\section{Literatur}

${ }^{1}$ Feigl GC, Ritz R, Moraes $M$ et al. Resection of malignant brain tumors in eloquent cortical areas: a new multimodal approach combining 5-aminolevulinic acid and intraoperative monitoring. J Neurosurg 2010; 113: 352-357

2 Feigl GC, Safavi-Abbasi S, Gharabaghi A et al. Real-time 3T fMRI data of brain tumour patients for intra-operative localization of primary motor areas. Eur J Surg Oncol 2008; 34: 708-715

${ }^{3}$ Kim HJ, Jeon BS, Paek SH. Effect of deep brain stimulation on pain in Parkinson disease. J Neurol Sci [Epub ahead of print]

${ }^{4}$ Kuhnt D, Ganslandt O, Schlaffer SM et al. Quantification of glioma removal by intraoperative high-field magnetic resonance imaging - an update. Neurosurgery [Epub ahead of print]

5 Moiyadi A, Shetty P. Objective assessment of utility of intraoperative ultrasound in resection of central nervous system tumors: a cost-effective tool for intraoperative navigation in neurosurgery. J Neurosci Rural Pract 2011; 2: 4-11

${ }^{6}$ Muns A, Meixensberger J, Arnold S et al. Integration of a 3D ultrasound probe into neuronavigation. Acta Neurochir (Wien) 2011; 153: 1529-1533

7 Voges J, Koulousakis A, Sturm V. Deep brain stimulation for Parkinson's disease. Acta Neurochir Suppl 2007; 97: 171-184

\section{Priv.-Doz. Dr. Dr. med. Günther C. Feigl} Leiter des Bereichs Intraoperative bildgebungsgestützte Neurochirurgie und Navigation

Prof. Dr. med. Marcos Tatagiba Direktor der Klinik für Neurochirurgie am Universitätsklinikum Tübingen

Klinik für Neurochirurgie am Universitätsklinikum Tübingen Hoppe-Seyler-Straße 3

72076 Tübingen

feigl@medigs.com 